\title{
Cell-to-Cell Communication within Intact Human Skin
}

Denis Salomon, Jean-Hilaire Saurat, and Paolo Meda *

Dermatology Clinic and *Institute of Histology, University of Geneva, Medical School, 1211 Geneva, Switzerland

\begin{abstract}
We have characterized cell-to-cell communication (coupling) within intact human skin by microinjecting single keratinocytes with a gap junction-permeant tracer (Lucifer Yellow). 25-50 keratinocytes from different layers of the epidermis were seen to be coupled after most injections $(n=31)$. A few noncommunicating cells were also microinjected $(n=3)$ or observed within large territories of coupled keratinocytes. Microinjections of dermal fibroblasts demonstrated an extensive coupling ( $>100$ fibroblasts); however, none of the keratinocyte $(n=34)$ or fibroblast $(n=3)$ injections revealed coupling between the epidermal and dermal compartments. Cell coupling was found to be more extensive in epidermal ridges than in suprapapillary plates and, in both regions, was less extensive after injection of the basal layer of the epidermis than after that of the suprabasal layers. This study shows that junctional cell-to-cell communications take place in normal and fully differentiated human tissue. The quantitative data gathered also indicate a regional heterogeneity of keratinocyte-to-keratinocyte communication within intact adult skin and the lack of effect of retinoids on this pattern.
\end{abstract}

\section{Introduction}

Like most other epithelial cells, the keratinocytes of human epidermis are connected by gap junctions $(1,2)$, i.e., by membrane differentiations thought to provide channels for direct cell-to-cell communication (3). Morphological reports of epidermal gap junction changes under a variety of pathological (4) and pharmacological conditions $(5,6)$ have suggested that junction-mediated cell communication, a phenomenon also referred to as cell coupling (3), is possibly involved in the functioning of keratinocytes. However, because the mere observation of gap junctions clearly does not imply that these structures are necessarily permeable $(7,8)$, it is still uncertain whether and how keratinocytes intercommunicate within the intact human skin. This tissue is quite different from newborn mice skin, in which cell coupling has recently been reported (9). Information about the normal pattern of junctional communication in intact human skin is required to assess further whether cell-to-cell communications change when the differentiation and/or proliferation program of keratinocytes is perturbed. As a first approach to this question, we have assessed quantitatively the extent and distribution of junctional com-

Address reprint requests to Dr. Denis Salomon, Clinique de Dermatologie, Hôpital Cantonal Universitaire, 1211 Geneva 4, Switzerland.

Received for publication 28 October 1987 and in revised form 21 January 1988.

J. Clin. Invest.

(C) The American Society for Clinical Investigation, Inc.

0021-9738/88/07/0248/07 \$2.00

Volume 82, July 1988, 248-254 munication between keratinocytes and between fibroblasts of human epidermis and dermis.

\section{Methods}

Skin samples. Keratome skin samples $\sim 180 \mu \mathrm{m}$ thick were obtained, while subjects were under anesthesia by intradermal injection of $1 \%$ xylocaine and $0.5 \mathrm{IU} / \mathrm{ml}$ Ornipressine (POR 8; Sandoz Ltd., Basel, Switzerland), from the buttocks of (a) seven healthy untreated volunteers; $(b)$ three volunteers treated topically with all-trans retinoic acid $(0.1 \%$ in $95 \%$ alcohol) once a day for $4 \mathrm{~d}$; $(c)$ one volunteer treated topically with etretin (Ro $10-1670 ; 0.1 \%$ in $100 \%$ alcohol and $10 \%$ dimethylsulfoxide), once a day for $4 \mathrm{~d}$; $(d)$ two patients that received 35 $\mu \mathrm{g} / \mathrm{d}$ arotinoid acid (Ro 13-7410) orally for $6 \mathrm{wk}$; and $(e)$ three untreated patients with psoriasis. In the latter two groups, skin was sampled from nonlesional regions.

Preparation of the tissue. Immediately after sampling, the skin was rinsed in PBS and transferred into a Krebs-Ringer medium buffered to pH 7.4 with $10 \mathrm{mM}$ Hepes and $25 \mathrm{mM} \mathrm{NaHCO}_{3}$ (control $\mathrm{KRB}$ ). Using a dissecting microscope, we cut each sample (usually $2 \times 10 \mathrm{~mm}$ ) into fragments of $1 \times 2 \mathrm{~mm}$ that were positioned, dermal side up, into 35-mm dishes (Falcon Labware, Becton, Dickinson, \& Co., Oxnard, CA) coated with Sylgard and $0.1 \%$ poly-L-lysine, as previously described (7). Each fragment was immediately covered with a drop of control KRB and allowed to attach to the dish for $5 \mathrm{~min}$. The dishes were then filled with $2 \mathrm{ml}$ of control KRB supplemented with $0.5 \%$ BSA ( $\mathrm{pH} 7.4$ ), and either immediately processed for microinjection or kept in an air $/ \mathrm{CO}_{2}$ incubator at $37^{\circ} \mathrm{C}$. The sampling and the preparation of the skin lasted $\sim 30 \mathrm{~min}$ and all fragments were microinjected and fixed within $3 \mathrm{~h}$ of surgery. This incubation time did not appear to affect the viability of the skin, as judged by the normal histological appearance of the fragments up to the end of the experiment, nor the extent of cell coupling. Indeed, uncoupled and coupled keratinocytes were observed both in fragments that were injected within half an hour of surgery and in those that were studied $2 \mathrm{~h}$ later.

Microinjection. The attached skin fragments were transferred to the heated $\left(37^{\circ} \mathrm{C}\right)$ stage of a Universal Zeiss microscope and were viewed from the top, i.e., from the dermal side, under dark-field illumination and at a magnification of 80 . Under continuous visual and electrophysiological control, a thin-tip glass microelectrode of $\sim 150 \mathrm{M} \Omega$ was lowered on the tissue. Because the microinjection of epidermal cells via the stratum corneum was not possible due to the hardness of this epidermal layer, skin fragments were attached with the stratum corneum lying against the bottom of the dish. In this arrangement, the electrode first encountered a small layer of dermis and, if pushed further down, entered the epidermis. Electrodes were filled with a $4 \%$ solution of Lucifer Yellow (10), in $150 \mathrm{mM} \mathrm{LiCl}_{2}$ buffered to $\mathrm{pH} 7.2$ with $10 \mathrm{mM}$ Hepes and connected to an electronic setup for the injection of current and the simultaneous recording of the electrode and cell potentials (7). After verification of the penetration of a cell by the sudden deflection of the recorded potential from 0 (electrode in the medium) to a negative resting membrane potential (electrode tip within a cell), Lucifer Yellow was injected ionophoretically by applying hyperpolarizing current pulses to the electrode, as described in reference 7. After $5 \mathrm{~min}$, the injected site was rapidly viewed under ultraviolet (UV) light and, whenever coupling was seen at this point, the injection was prolonged for 10 additional minutes. At the end of the injection period ( $5 \mathrm{~min}$ in case of uncoupling and $15 \mathrm{~min}$ in case of coupling), the electrode was pulled out of the cell (this was assessed by a 
suddeh return of the monitored potential toward the zero line) and the injected site was photographed under fluorescence illumination (7, 11). After removal of the medium, the skin fragment was fixed in the dish using a 4\% solution of paraformaldehyde in PBS, $\mathrm{pH} 7.4$ (7). The samples were then stored at $4^{\circ} \mathrm{C}$ until subsequent histological processing.

Light microscopy. The microinjected and fixed samples were dehydrated in alcohols and embedded either in araldite or Historesin (LKB Instruments, Gaithersburg, MD), according to standard procedures. $2-\mu \mathrm{m}$-thick serial sections, cut throughout each block perpendicularly to the stratum corneum, were scored whenever they contained Lucifer Yellow-labeled cells and photographed under a Zeiss photomicroscope using epifluorescence illumination. Some sections were immunostained further for keratins. To this end; the araldite was removed by applying a $\mathrm{NaOH} /$ methanol/propylene oxide mixture ( 1 $\mathrm{g}: 5 \mathrm{ml}: 5 \mathrm{ml}$ ) on the sections for $2.5 \mathrm{~min}$. The sections were rinsed in methanol, then in PBS, and finally incubated with $0.25 \%$ trypsin (porcine type II; Sigma Chemical Co., St. Louis, MO) for $18 \mathrm{~min}$ at $37^{\circ} \mathrm{C}$ (12). After being rinsed in PBS, the sections were incubated with an anti-keratin rabbit polyclonal antibody (diluted 1:20; DAKOPATTS, Copenhagen, Denmark) for $60 \mathrm{~min}$ at room temperature. A second incubation was then performed by applying a rhodamine-conjugated anti-rabbit IgG (diluted 1:20; Cappel Laboratories, Cochranville, PA) for $60 \mathrm{~min}$ at room temperature. The immunostained sections were finally coverslipped in $98 \%$ glycerol for fluorescence photography.

Quantitative analysis. Quantitative analysis of cell coupling was performed on color slides of all the serial sections that contained cells labeled by Lucifer Yellow. These slides were projected at a final magnification of 500. For each injection site, coupling was evaluated by three parameters: (a) the spatial length (in micrometers) of cell-to-cell communication, which was calculated by multiplying the total number of sections containing Lucifer Yellow-labeled cells by the section thickness $(2 \mu \mathrm{m}) ;(b)$ the total number of coupled cells per injection, which was obtained by scoring the keratinocytes labeled by Lucifer Yellow on serial sections taken at an interval of $14 \mu \mathrm{m}$, representing the average diameter of a basal keratinocyte; $(c)$ the surface (squared micrometers) occupied by the coupled cells, which was measured on the same sections using a graphics tablet connected to a personal computer programmed for area measurements (7). Since the values of each of the three parameters studied showed a nonnormal distribution (Fig. 4), average values were expressed as medians and, accordingly, were compared using the median test (13). The distributions of the three parameters evaluated were also compared using the KolmogorovSmirnov and the Mann-Whitney tests (13).

\section{Results}

Qualitative observation. Fig. $1 \mathrm{~A}$ shows the typical appearance of a skin fragment as seen from the dermal side, in the microinjection setup. Under dark-field illumination, each fragment appeared to be formed by circular dark areas that corresponded in size, shape, and distribution to the dermal papillae seen in perpendicular sections of the keratome samples (Fig. 1 $B)$. These papillae were surrounded by lighter bands of a more homogeneous tissue (Fig. $1 A$ ) that correspond to the epidermal ridges seen on sections (Fig. $1 B$ ). By positioning the electrode tip over either the round dark areas or the lighter homogeneous bands, microinjections thus could be performed in either the dermal or the epidermal compartment. When a dermal cell was injected $(n=3)$, Lucifer Yellow rapidly diffused into a large number of nearby cells that, in the intact tissue and at the low magnification used during microinjection, were seen as groups of fluorescent spots within adjacent dermal papillae (Fig. $2 A$ ). In comparison, when an epidermal cell was injected $(n=34)$, Lucifer Yellow labeled either only the site of
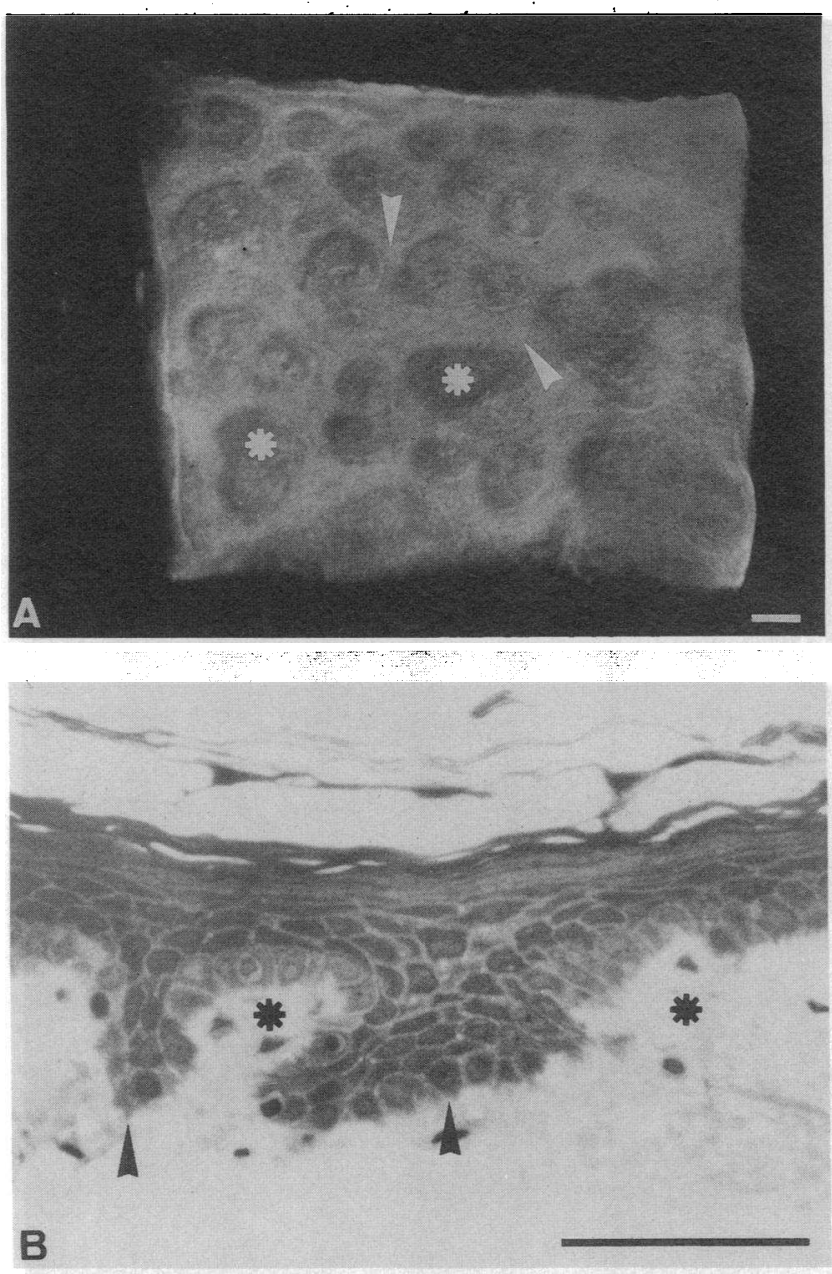

Figure 1. Appearance of the keratome skin fragments. $(A)$ Under the dark-field microscope that was used for microinjection, a fragment shows several dermal papillae (asterisks) surrounded by epidermal ridges (arrowheads). (B) These two regions are clearly distinguished on a perpendicular section of a fragment that was fixed and processed for histology at the end of the microinjection. Sections also demonstrated the normal appearance of the skin and showed that the thickness of each fragment was adjusted so as to sample all epidermal layers with as little connective dermal tissue as possible. Bar, $50 \mu \mathrm{m}$ in $A$ and $B$.

injection (not shown) or a larger area (Fig. 2 B). In the latter case, the injected tissue was homogeneously stained by Lucifer Yellow and showed the stellate appearance of epidermal ridges (Fig. 2 B).

In view of the tridimensional organization of the skin fragments, the location, arrangement, and type of communicating cells could not be assessed directly during the injection and was determined, a posteriori, on serial sections that were cut throughout each skin sample. Analysis of these sections revealed that the pattern of fluorescence seen after injection of a dermal papilla was due to the labeling of a large number (usually $>100$ ) of triangular or spindle-shaped cells that were separated from each other by unstained areas, except at the extremity of thin processes, and were identified as fibroblasts (Fig. $2 C$ ). In comparison, sections demonstrated that the pattern of fluorescence seen after injection of an epidermal ridge was due to the presence of Lucifer Yellow within polygonally 

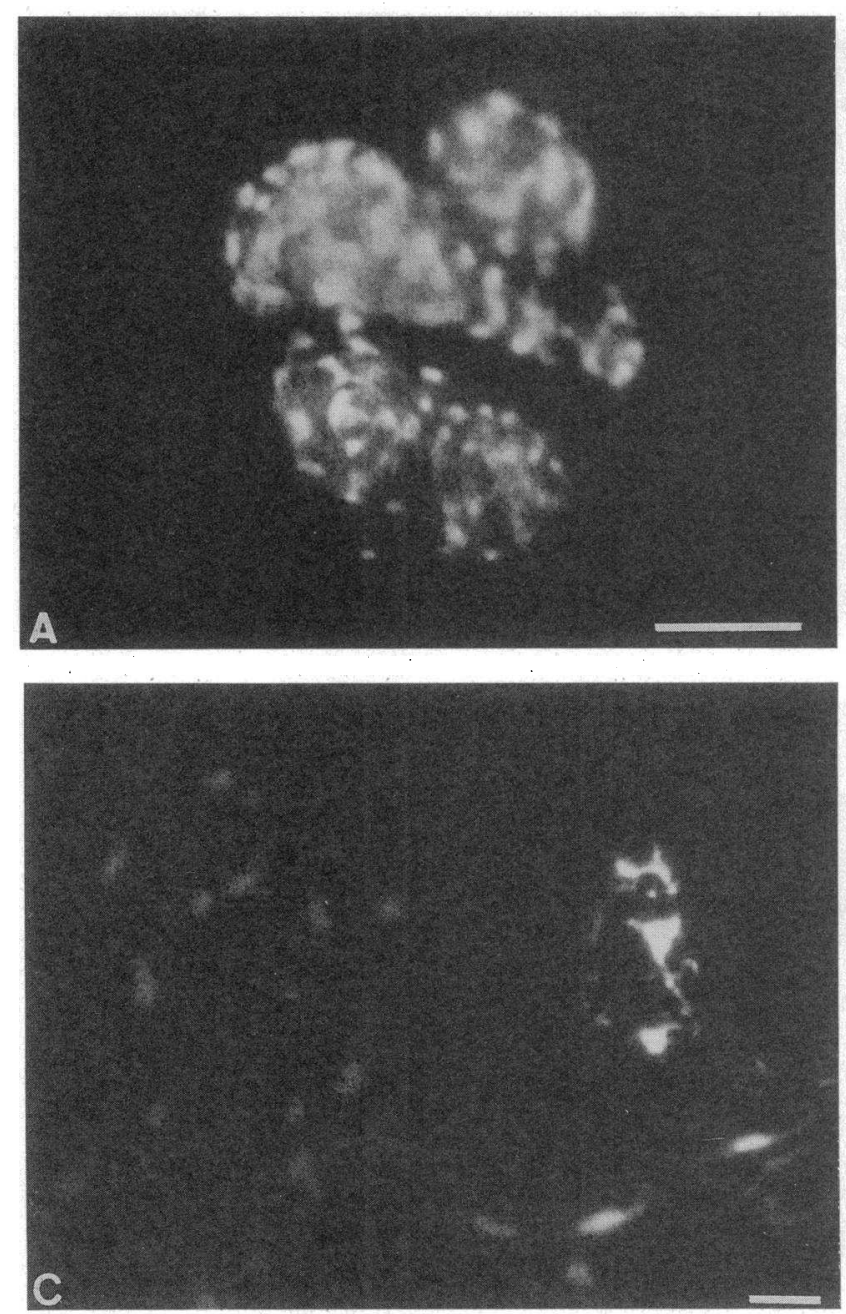

Figure 2. Fluorescence views of intact skin fragments injected with Lucifer Yellow $(A$ and $B$ ) and of corresponding representative sections $(C$ and $D)$. $(A)$ After microinjection of a dermal cell, the spotty pattern of fluorescence within circular domains indicates the transfer of Lucifer Yellow between fibroblasts of adjacent papillae. (B) After microinjection of an epidermal cell, the homogeneous pattern of fluorescence indicates the labeling of adjacent keratinocytes within an epidermal ridge. $(C)$ Sectioning of a communication territory resulting from the microinjection of a dermal cell confirmed the extensive coupling of spindle-shaped fibroblasts. Note the absence of fluores-

shaped cells that were in close contact and showed the characteristic morphology of keratinocytes (Fig. $2 \mathrm{D}$ ).

In most cases, the microinjected keratinocyte was identified both in fragments and sections by a more intense labeling than that seen in nearby cells. A gradient of fluorescence was seen running from the injected cell through the neighbor coupled keratinocytes (Figs. $2 D$ and $3 A$ ). Often, the epidermal territories labeled by Lucifer Yellow comprised both basal and suprabasal keratinocytes (Figs. $2 D$ and $3 A$ ) and a number of unlabeled, apparently uncoupled cells (Fig. $3 \mathrm{~A}$ ). Immunolabeling with an anti-keratin antibody confirmed that the epidermal coupled cells were keratinocytes from basal and suprabasal layers (Fig. $3 \mathrm{~B}$ ). In comparison, most of the cells that were not labeled by Lucifer Yellow were also keratin-negative (Fig. $3 \mathrm{~B}$ ). These cells were therefore considered tentatively
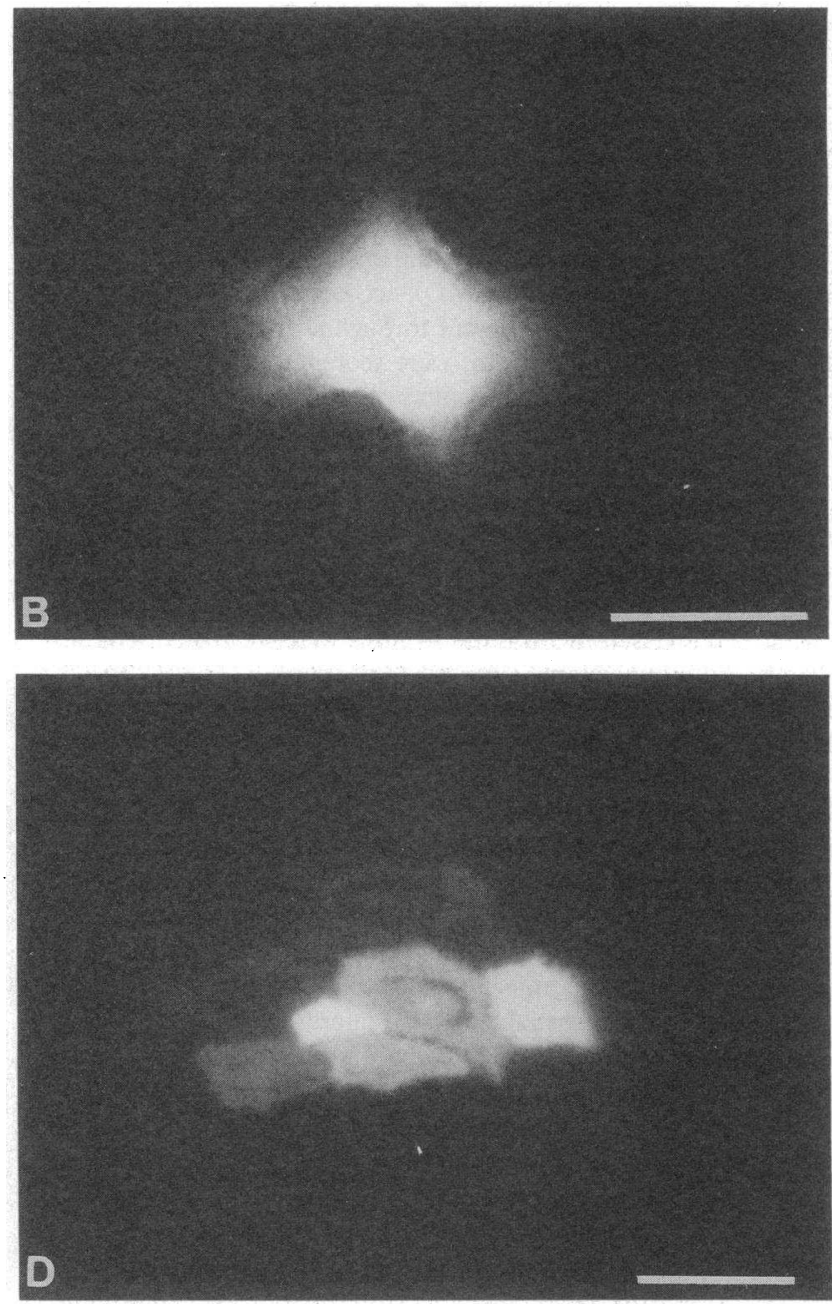

cence labeling of nearby epithelial cells. $(D)$ In comparison, microinjection of an epidermal cell led to the labeling of a smaller number of polygonally shaped keratinocytes in close contact. In this case, the microinjected cell, which is identified by a very intense fluorescence, was a suprabasal keratinocyte and the nearby coupled keratinocytes were located in both basal and suprabasal layers of the epidermis. Note the decrease of the gradient of fluorescence from the injected cell through the keratinocytes located at the periphery of the communication territory and the absence of Lucifer Yellow transfer to fibroblasts. Bar, $50 \mu \mathrm{m}$ in $A$ and $B$ and $15 \mu \mathrm{m}$ in $C$ and $D$.

either melanocytes, if they were found in the basal layer of the epidermis, or as Langerhans cells and/or white blood cells, if they were found throughout the epidermis (Fig. $3 B$ ).

No instance of Lucifer Yellow transfer into the dermal compartment of the skin was observed after epidermal microinjections. Similarly, the tracer did not diffuse into the epidermis when microinjections were performed in a dermal cell.

Light microscopy screening of serial sections (Fig. $1 B$ ) showed the normal histological appearance of skin fragments up to the end of the 3-h microinjection experiments and the complete absence of skin appendages, such as hair follicles or glands, in the relatively thin keratome samples we used. Qualitative examination indicated that both basal and suprabasal keratinocytes within epidermal papillae and ridges had a similar size and shape and did not reveal major alterations of kera- 

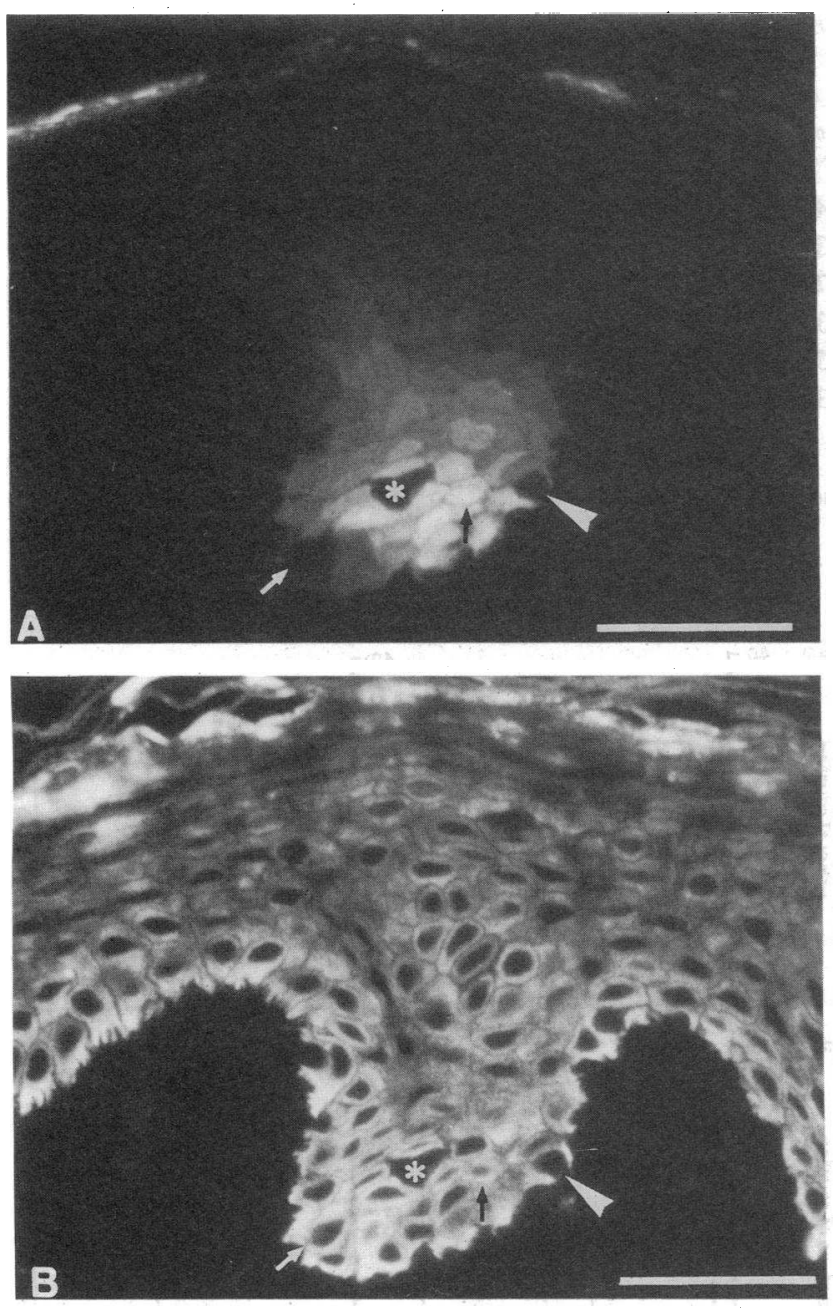

Figure 3. Fluorescence and immunolabeling of an epidermal territory of coupled keratinocytes. $(A)$ The fluorescence view permits one to determine the location of the injected cell (black arrow) in the lower layers of the epidermis and its communication with a large number of both basal and suprabasal keratinocytes. Note the presence, within the communication territory, of several uncoupled cells (asterisk, arrow, arrowhead). (B) After incubation with an anti-keratin antibody, most of the coupled cells are immunostained and thus are positively identified as keratinocytes. Two of the uncoupled cells are keratin-negative (asterisk, arrowhead) and in view of their shape and position, may represent a Langerhans cell (asterisk) and a melanocyte (arrowhead). The white arrow points to an immunolabeled basal keratinocyte that was uncoupled. Bar, $40 \mu \mathrm{m}$ in $A$ and $B$.

tinocyte morphology after treatment with retinoids. The sections that were cut perpendicular to the stratum corneum to prevent the tangential sectioning of epidermal ridges (Figs. 1 and 3), were also instrumental in differentiating basal keratinocytes that were in contact with the basement membrane zone from the morphologically different suprabasal keratinocytes (14).

Quantitative analysis. To evaluate the coupling of keratinocytes more precisely, we quantitated the spatial extent and the area of each communication territory and the total number of coupled cells per injection. Regression analysis indicated a significant $(P<0.001)$ correlation between the values of the three parameters that were measured independently. Scatter-

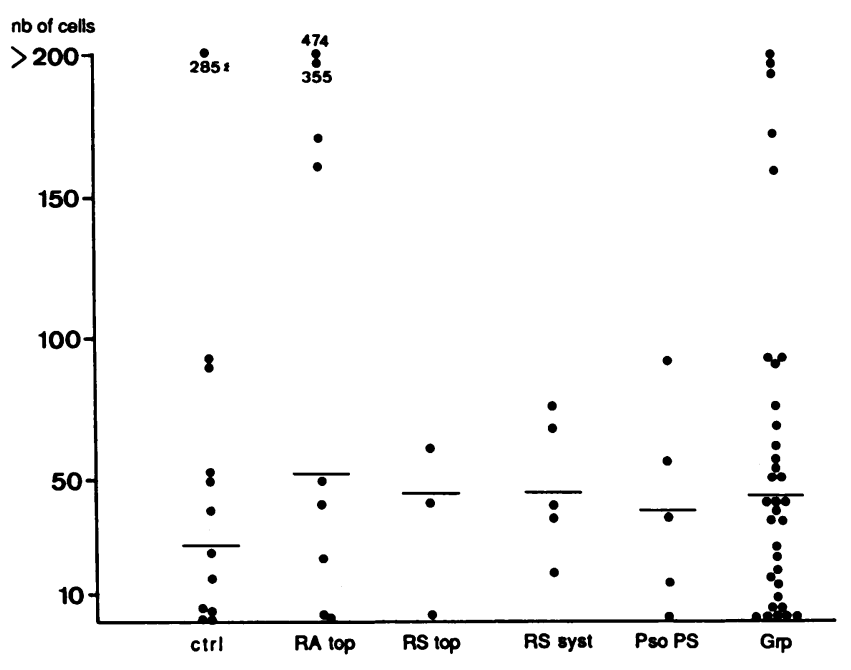

Figure 4. Scattergram of the number of coupled keratinocytes per injection. The bar presents the median value of each group. Abbreviations: ctrl, control; RA top, topical retinoic acid; RS top, topical synthetic retinoid; RS syst, systemic synthetic retinoid; Pso PS, uninvolved psoriatic skin; and Grp, values pooled from all groups.

grams (Fig. 4 shows the number of coupled cells per injection) revealed a skewed distribution of the three parameters evaluated. Accordingly, the average values of these parameters were expressed as medians. In control skin $(n=12)$, the median value of the extent of coupling, the surface of coupling, and the number of coupled cells were $23 \mu \mathrm{m}, 1,089 \mu \mathrm{m}^{2}$, and 26 cells, respectively (Table I). These values were not significantly decreased in normal skin treated with retinoids either topically or systemically ${ }^{1}$ and in uninvolved psoriatic skin (Table I). Furthermore, when the data from the treated groups were pooled and compared with controls, no significant difference was detected (not shown). The values from these groups, which were all obtained from the same site of clinically and histologically normal skin, thus were pooled to determine whether keratinocyte coupling varies in different regions of the epidermis. Therefore, the pooled values of the three parameters evaluated were computed separately, first for keratinocytes injected in suprapapillary plates (the region of the epidermis above dermal papillae) and ridges (the region of the epidermis extending between dermal papillae) and, second, for keratinocytes injected in the basal and suprabasal layers of the epidermis. As shown in Fig. 5, the spatial extent and the area of intercellular communication, as well as the number of coupled cells, were significantly $(P<0.04-0.005)$ higher when microinjections were performed within an epidermal ridge than when injections were performed within a suprapillary plate. Statistical analysis also revealed a significantly $(P<0.002)$ larger coupling after injection of suprabasal than of basal keratinocytes (Fig. 5).

\section{Discussion}

We provide here the first report on the pattern and regional organization of junctional communication between keratino-

1. Both topical and systemic retinoid treatments significantly increased $(P<0.0025)$ the epidermal levels of cytosolic retinoid acid-binding proteins in the patients from whom keratome samples were obtained for microinjection $(32,33)$. 
Table I. Quantitative Evaluation of Keratinocyte Coupling in Intact Human Skin

\begin{tabular}{|c|c|c|c|c|c|c|}
\hline & Control & $\begin{array}{l}\text { Topical } \\
\text { retinoic acid }\end{array}$ & Topical retinoid & Systemic retinoid & $\begin{array}{l}\text { Uninvolved } \\
\text { psoriatic skin }\end{array}$ & Pooled values \\
\hline \multirow[t]{2}{*}{ Extent of coupling $(\mu \mathrm{m})$} & 23 & 30.5 & 24 & 26 & 30 & 26.5 \\
\hline & $(12)$ & (9) & (3) & (5) & (5) & $(34)$ \\
\hline \multirow[t]{2}{*}{ Surface of coupling $\left(\mu \mathrm{m}^{2}\right)$} & 1,089 & 1,678 & 927 & 1,615 & 636 & 1,256 \\
\hline & (11) & (9) & (3) & (5) & (4) & (32) \\
\hline \multirow[t]{2}{*}{ No. of coupled cells } & 25.5 & 49 & 43 & 41 & 36 & 41.5 \\
\hline & $(12)$ & (9) & (3) & (5) & (4) & (33) \\
\hline
\end{tabular}

Values are medians of the number of injections indicated in parentheses. The topical retinoid was etretin (Ro 10-1670), the systemic retinoid was arotinoid acid (Ro 13-7410).

cytes of adult human skin. This study extends previous observations on the electrical (15) and metabolic coupling of human keratinocytes in culture (16) and in mechanically separated epidermis (17) by demonstrating intercellular communication in the normal intact tissue. By using the intracellular microinjection of a membrane-impermeant but gap junction-permeant tracer, coupled cells were evaluated quantitatively and their organization characterized in different regions of the dermis and epidermis. In a complex heterocellular and tridimensional system like skin, this approach provides a more direct assessment of junctional communication than electrophysiological measurements $(18,19)$. Recently, a similar strategy was adopted to study coupling in newborn mouse skin (9), a tissue that differs markedly from adult human skin from both the developmental and the histological standpoint.

The three main observations of our study can be summarized as follows. First, cell-to-cell communication occurs in both the dermal and epidermal compartments of normal and fully differentiated human skin. In the dermis, coupling usually interconnected several hundred fibroblasts from adjacent papillae. In comparison, in the epidermis, coupling was more restricted, as it usually involved only a few dozen keratinocytes. Occasionally, cells that did not appear to be coupled were observed among the communicating keratinocytes. These cells were not positively identified, but their lack of immunolabeling for keratins and their distribution in the tissue suggest that they were mostly melanocytes, Langerhans cells, or transiting blood cells. In the intact epidermis, coupling thus occurs mostly between keratinocytes, even though infrequent communications may also take place between heterologous skin cells in culture, as shown autoradiographically (19, 20). It has been claimed recently that heterocellular communications also occur between keratinocytes and fibroblasts of neonatal mouse skin (9). We have not observed such communication in the adult human skin. Thus, in no case was Lucifer Yellow exchanged between the dermal and the epidermal compartments of this tissue, even though a number of our injections led to extensive coupling of either fibroblasts or keratinocytes located very close to the basal membrane that normally separates the two skin compartments. These contrasting observations may be explained by the different species, developmental stages, and histology of the two skins studied. In the study on mice, the neonatal skin that was injected was formed by a thin epidermis of three to five layers and a much thicker dermis cofltaining glands and developing hair follicles (9),
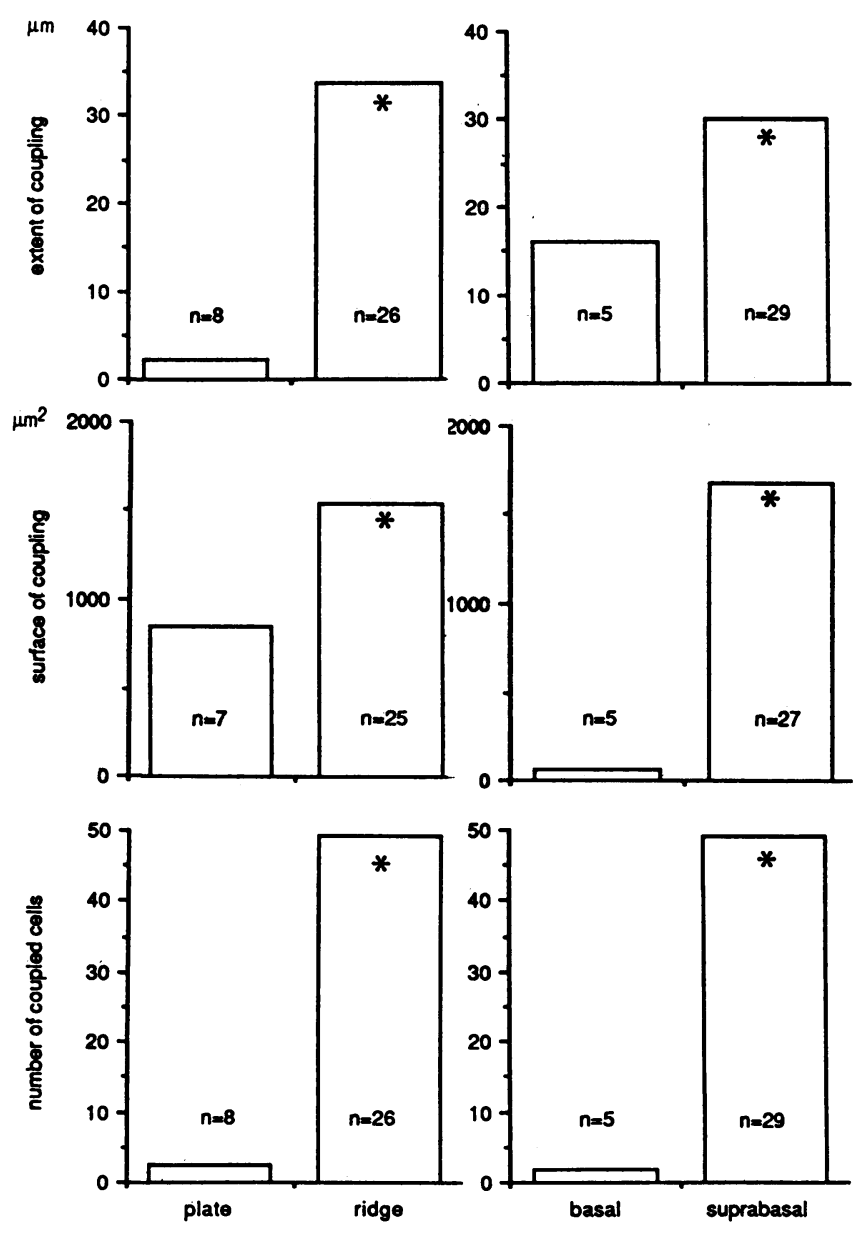

Figure 5. Quantitative evaluation of keratinocyte coupling within different regions of intact human epidermis. The upper, middle, and lower panels show the median value of the extent of coupling, the area of coupling, and the number of coupled cells, respectively. Comparison of these three parameters using the median test, showed a significant $(P<0.04-0.005)$ difference (asterisk) between the values evaluated in suprapapillary plates and in epidermal ridges. Similarly, the values characterizing coupling were significantly ( $P$ $<0.04-0.005)$ higher when Lucifer Yellow injections were performed in a suprabasal rather than in a basal keratinocyte. Comparison of the distributions of the three parameters tested, using either the Kolmogorow-Smirnov or the Mann-Whitney test, also showed significant $(P<0.02-0.001)$ differences between plates and ridges and between basal and suprabasal layers (not shown). 
whereas in our experiments the adult human skin studied was formed mostly by a thick epidermis and a minimal amount of dermis lacking skin appendages. The latter structures, which are located deeply in the dermis and were not included in our superficial keratome samples, may be the sites at which dermal-epidermal communications take place (9). Alternatively, epidermal-dermal communication may result from the successive penetration of the injecting electrode into different cells of the two skin compartments. This possibility cannot be ruled out, because, at variance with our protocol, previous experiments were not performed under continuous electrophysiological monitoring that would allow one to control for such multiple cell penetrations within the tridimensional skin fragments $(9,21)$.

The second set of information is provided by the quantitative evaluation of keratinocyte coupling in different regions of the epidermis. Most often, keratinocytes exchanged Lucifer Yellow throughout the different layers of the epidermis, except the stratum corneum. This pattern was seen irrespective of whether the tracer had been introduced in a basal or suprabasal keratinocyte indicating that, in intact skin, coupling actually establishes a functional interconnection of cells at very diverse stages of differentiation. In view of the various characteristics of keratinocytes in the different layers $(14,22,23)$ and regions of the epidermis (24), we have further investigated whether coupling is regionally heterogeneous. Our analysis has revealed that junctional communication is more extensive after injection of suprabasal than of basal keratinocytes, a finding consistent with the predominant distribution of gap junctions in the upper layers of the epidermis (2). From both a functional and a structural standpoint, the transmission of information through permeable junctions thus appears to be easier from the more differentiated compartment of the epidermis toward the proliferative compartment of the tissue, than vice versa. Coupling was found to be also much greater between the keratinocytes of the epidermal ridges than between those of the suprapillary plates. These regional differences indicate that the ability of keratinocytes to communicate with nearby companion cells is somehow dependent on their state of differentiation, cellular and noncellular environment, and dividing capacity, all of which vary as a function of the cells' anatomical location (14, 22-24). The alternative possibility, that the coupling differences we observed between epidermal plates and ridges simply reflected differences in the size, shape, or volume (25) of otherwise analogous keratinocytes, is unlikely, since histology did not reveal major differences in these cell parameters between the two epidermal regions.

Finally, in view of the apparently contradictory effects of retinoids on gap junctions and cell coupling in different systems (26-29), and of the importance of these compounds in the regulation of both normal and pathological biology of the skin $(30,31)$, we have studied the effects of local and systemic retinoid treatments on keratinocyte coupling. As reported, the two treatments studied did not decrease the exchange of Lucifer Yellow between keratinocytes, under conditions that led to a significant increase in the epidermal levels of cytosolic retinoic acid binding proteins, i.e., that biologically affected the skin $(32,33)$. Since some retinoid treatments have been reported to affect keratinocyte structure (34), a change that could conceivably influence cell coupling or, at least, its detection by the dye injection approach (25), we had to consider the possibility that our analysis had failed to detect a subtle but significant effect of retinoids on the total volume of coupled keratinocytes. Although our data do not allow us to totally exclude this possibility, we consider it rather unlikely, again in view of the lack of evident keratinocyte changes that was documented histologically at the end of both the topical and systemic treatment we tested. At any rate, these retinoid treatments did not uncouple human keratinocytes. This conclusion agrees with previous reports that retinoids do not modify coupling (28) and do not prevent reestablishment of gap junctions between homologous cells (27), but contrasts with other observations that have indicated a blocking effect of retinoids on the junctional communication of several cell types $(26,29)$. Whether these differences outline the variable regulation of gap junctions in different systems (35) or are merely related to differences in the dose ${ }^{2}$ or type of the retinoids tested, remains to be established.

In summary, we have shown that junctional cell-to-cell communications take place in normal and fully differentiated adult human tissue and can be studied quantitatively without perturbing the normal organization of the intact organ. The possible function(s) of junctional communications between adult keratinocytes remain(s) to be established, as is true for the role of junctional coupling in most other nonexcitable cell systems $(35,36)$. Increasing circumstantial evidence points to a likely role of gap junctional communication in the control of general cell functions such as growth, differentiation, and metabolism $(3,30,35)$, and in the modulation of more specific activities of adult differentiated cells, such as secretion (36). Whether any of these putative functions are relevant to the biology of human keratinocytes can be addressed using the model and approach we have outlined here. Thus, the comparison of coupling in control and pathological skin, under conditions perturbing the normal pattern of keratinocyte multiplication, differentiation, and/or metabolism $(4,37)$, should provide insights into the possible role of junctional communications in the functioning of skin cells. In some pathological conditions, ultrastructural studies have indeed suggested alterations of keratinocyte gap junctions $(6,37)$.

\section{Acknowledgments}

We thank D. Grand, A. Charollais, P. Ruga, and P. Sardy for technical assistance; S. Deschamps for skillful secretarial help; and Dr. S. Hirschel-Scholz for providing some of the skin samples.

This work was supported by grants from the Swiss National Science Foundation (3.404.86) and the National Institutes of Health (5RO1AM-30519-02), and by a grant-in-aid from the Sociéte Académique de Genève.

\section{References}

1. Breathnach, A. S., C. Stolinski, and M. Gross. 1972. Ultrastructure of foetal and postnatal human skin as revealed by the freeze-fracture replication technique. Micron. 3:287-304.

2. In several culture systems $(28,31)$ cell uncoupling has been reported after exposure to concentrations $\left(\sim 10^{-4} \mathrm{M}\right)$ of retinoids that are toxic for rat liver epithelial cells (28), and that are much higher than the pharmacologically active concentrations of these compounds $\left(10^{-6}\right.$ $10^{-9} \mathrm{M}$ ) that we tested. In preliminary in vitro studies we also observed that the electrical (voltage clamp technique) and dye coupling of human keratinocytes may be rapidly abolished by $10^{-4} \mathrm{M}$, but not by $10^{-7}$ and $10^{-6} \mathrm{M}$ all-trans retinoic acid (unpublished). 
2. Caputo, R., and D. Peluchetti. 1976. The junctions of normal human epidermis. A freeze-fracture study. J. Ultrastruct. Res. 61:4461 .

3. Loewenstein, W. R. 1981. Junctional intercellular communication: the cell-to-cell membrane channel. Physiol. Rev. 61:830-913.

4. Caputo, R., and G. Gasparini. 1982. Freeze-fracture replication technique of human skin. J. Cutaneous Pathol. 9:203-224.

5. Elias, P. M., and D. S. Friend. 1976. Vitamin-A-induced mucous metaplasia: an in vitro system for modulating tight and gap junction differentiation. J. Cell Biol. 68:173-188.

6. Elias, P. M., S. Grayson, T. M. Caldwell, and N. S. McNutt. 1980. Gap junction proliferation in retinoic acid-treated human basal cell carcinoma. Lab. Invest. 42:469-474.

7. Meda, P., R. Bruzzone, S. Knodel, and L. Orci. 1986. Blockage of cell-to-cell communication within pancreatic acini is associated with increased basal release of amylase. J. Cell Biol. 103:475-483.

8. Miller, T., and D. Goodenough. 1985. Gap junction structures after experimental alteration of junctional channel conductance. $J$. Cell Biol. 101:1741-1748.

9. Kam, E., L. Melville, and J. D. Pitts. 1986. Patterns of junctional communication in skin. J. Invest. Dermatol. 87:748-753.

10. Stewart, W. W. 1978. Functional connections between cells as revealed by dye-coupling with a highly fluorescent naphthalimide tracer. Cell. 14:741-759.

11. Meda, P., R. M. Santos, and I. Atwater. 1986. Direct identification of electrophysiologically monitored cells within intact mouse islets of Langerhans. Diabetes. 35:232-236.

12. Pinkus, G. S., E. M. O'Connor, C. L. Etheridge, and J. M. Corson. 1985. Optimal immunoreactivity of keratin study of various tumors using polyclonal and monoclonal antibodies. J. Histochem. Cytochem. 33:465-473.

13. Siegel, S. 1956. Nonparametric statistics for the behavioral sciences. McGraw-Hill Book Co., Tokyo. 47-52.

14. Holbrook, K. A., and K. Wolff. 1987. The structure and development of skin. In Dermatology in General Medicine. T. B. Fitzpatrick, A. Z. Eisen, K. Wolff, I. M. Freedberg, and K. W. Austen, editors. Vol. 9. McGraw-Hill Book Co., New York. 93-131.

15. Cavoto, F. V., and B. A. Flaxman. 1972. Communication between normal human epidermal cells in vitro. $J$. Invest. Dermatol. 59:370-374.

16. McKay, I., and J. Taylor-Papadimitriou. 1982. The nonselective junctional communication phenotype of normal and transformed human epidermal keratinocytes in vitro. Exp. Cell Res. 141:171-180.

17. Van Heukelom, J. S., D. W. Slaaf, and J. C. Van Der Leun. 1972. Cell communication in the basal cells of the human epidermis. Biophys. J. 12:1266-1284.

18. Socolar, S. J., and W. R. Loewenstein. 1979. Methods for studying transmission through permeable cell-to-cell junctions. Methods Membr. Biol. 10:121-177.

19. Meda, P. 1987. Fluorescence in the study of direct intercellular communications: the case of pancreatic cells. In Microspectrofluorimetry of Single Cells. E. Kohen, editor. Academic Press, New York. In press.
20. Hunter, K., and J. D. Pitts. 1981. Non-selective junctional communication between some different mammalian cell types in primary culture. J. Cell Sci. 49:163-175.

21. Pitts, J., E. Kam, L. Melville, and F. M. Watt. 1986. Patterns of junctional communication in animal tissues. Ciba Found. Symp. 125:140-153.

22. Green, H. 1979-1980. The keratinocyte as differentiated cell type. Harvey Lect. 74:101-139.

23. Doran, T. I., A. Vidrich, and T.-T. Sun. 1980. Intrinsic and extrinsic regulation of the differentiation of skin, corneal and esophageal epithelial cells. Cell. 22:17-25.

24. Lavker, R. L., and T.-T. Sun. 1982. Heterogeneity in epidermal basal keratinocytes: morphological and functional correlations. Science (Wash. DC). 215:1239-1241.

25. Sheridan, J. D. 1973. Functional evaluation of low resistance junctions: influence of cell shape and size. Am. Zool. 13:1119-1129.

26. Pitts, J. D., A. E. Hamilton, E. Kam, R. R. Burk, and J. P. Murphy. 1986. Retinoic acid inhibits junctional communication between animal cells. Carcinogenesis (Lond.). 7:1003-1010.

27. Chertow, B. S., N. G. Baranetsky, W. I. Sivitz, P. Meda, M. D. Webb, and J. C. Shih. 1983. Cellular mechanisms of insulin release. Effects of retinoids on rat islet cell-to-cell adhesion, reaggregation, and insulin release. Diabetes. 32:568-574.

28. Mehta, P. P., J. S. Bertram, and W. R. Loewenstein. 1986. Growth inhibition of transformed cells correlates with their junctional communication with normal cells. Cell. 44:187-196.

29. Walder, L., and R. Lutzelschwab. 1984. Effects of 12-O-tetradecanoylphorbol-13-acetate (TPA), retinoic acid and diazepam on intercellular communication in a monolayer of rat liver epithelial cells. Exp. Cell Res. 152:66-76.

30. Fuchs, E., and H. Green. 1981. Regulation of terminal differentiation of cultured human keratinocytes by vitamin A. Cell. 25:617-625.

31. Saurat, J. H. 1985. Retinoids: New Trends in Research and Therapy. Karger, Basel, Switzerland. 514 pp.

32. Siegenthaler, G., and J.-H. Saurat. 1986. Therapy with a synthetic retinoid-(Ro-1670) Etretin-increases the cellular retinoic acid-binding protein in nonlesional psoriatic skin. J. Invest. Dermatol. 87:122-124.

33. Hirschel-Scholz, S., G. Siegenthaler, and J.-H. Saurat. 1987. Increase of human epidermal CRABP after topical application of etretin and retinoic acid. J. Invest. Dermatol. 89:311 (Abstr.)

34. Elias, P. M., and M. L. Williams. 1985. Retinoid effects on epidermal differentiation. In Retinoids: New Trends in Research and Therapy. J. H. Saurat, editor. Karger, Basel, Switzerland. 138-158.

35. Hertzberg, E. L., T. S. Lawrence, and N. B. Gilula. 1981. Gap junctional communication. Annu. Rev. Physiol. 43:479-491.

36. Meda, P., A. Perrelet, and L. Orci. 1984. Gap junctions and cell-to-cell coupling in endocrine glands. Mod. Cell Biol. 3:131-196.

37. Caputo, R., M. Innocenti, G. Gasparini, and D. Peluchetti. 1978. Plasma membranes in psoriatic cells. A freeze-fracture study. $J$. Invest. Dermatol. 71:245-249. 